\title{
A REGRESSION MODEL OF DRY MATTER ACCUMULATION FOR SOLAR GREENHOUSE CUCUMBER
}

\author{
Weitang Song ${ }^{1,2,4}$, Xiaojun Qiao ${ }^{3}$ \\ ${ }^{1}$ College of Science, China Agricultural University, Beijing, China, 100094 \\ ${ }^{2}$ Key Lab in Bioenvironmental Engineering of Ministry of Agriculture, China Agricultural \\ University, Beijing, China, 100083 \\ ${ }^{3}$ National Engineering Research Center for Information Technology in Agriculture, Beijing, \\ China, 100097 \\ ${ }^{4}$ Corresponding author, Address: Institute of Agricultural Engineering, China Agricultural \\ University, 2 Yuanmingyuan West Road, Beijing, 100094, P. R. China, Email: \\ songchali@cau.edu.cn
}

Abstract: The objective of this study is to develop a regression cucumber dry matter production model with a minimum number of parameters. Cucumber (Cucumis sativus L.) was cultivated in a soilless system with drip irrigation. The substrate was peat mixed with vermiculite. Five experiments were fulfilled totally in 3 different places in Beijing of China from 2004 to 2005. Cucumber growth data (dry matter weight of leaf, stem, fruit and petiole) were measured and environmental data (temperature, light intensity and day length) were collected. Data collected from 1 experiment in solar greenhouse was used to build the model, which was further validated with the data collected from other 4 experiments in solar greenhouse. A regression model for cucumber dry matter production was established. Based on Logistic curve, the time state variable was expressed as a logistic function about effective temperature accumulation (ETA) and effective light intensity accumulation (ELIA). ETA was defined as the sum of the temperature that was higher than physiological zero point in certain period, and ELIA was defined as the sum of the light intensity that was higher than light compensation point multiplied with time in certain period. Temperature, light intensity and day length were synthetically considered. The model had less state variables, and provided the relationships between the cucumber dry matter accumulation (DMA) per plant and environmental data (temperature, radiation and day length). The result of simulation was satisfied, because RMSE value was less than 6 , and the $\mathrm{R}^{2}$ value of the results was 0.99 . It indicated that the regression model for cucumber dry matter production was reasonable and feasible.

Song, W. and Qiao, X., 2008, in IFIP International Federation for Information Processing, Volume 259; Computer and Computing Technologies in Agriculture, Vol. 2; Daoliang Li; (Boston: Springer), pp. 1333-1339. 
Keywords: cucumber, dry matter accumulation, regression model, effective temperature accumulation, effective light intensity accumulation

\section{INTRODUCTION}

Both mechanism models and regression models can be used to describe the course of cucumber dry matter accumulation (DMA) (Challa et al., 1996; Heuvelink et al., 1996). Mechanism models generally have many submodels for each DMA process, which can describe crop's growing course and have strong explanation function completely (Li et al., 1999). But regression models show directly the relationship between crop development, growth rate, output and environmental factors by functions of multivariate regression equation, index equation, hyperbola equation and "s" curve, etc. It describes the results of observation.

Most present cucumber dry matter accumulation models are explanatory. The application limitations of these models focus on that: (1) they have so many parameters that it is very complicated to be calculated (Challa et al., 1996); (2) they need to input many variables and data, but the output is wavy; and (3) the prediction is not very accurate. Contrarily, regression model parameters are easier to estimate and the calculation process is short, which is practical in real time control for greenhouse.

The accumulation course of dry matter mainly includes photosynthesis and respiration. Temperature and light are two most important factors of cucumber DMA, if nutrition and water are sufficient and plants don't have pests (Chamont et al., 1993). A lot of models describe crop growth with temperature accumulation or effective temperature accumulation (ETA) (Katharine, 1996). Illumination is also important to influence crop growth, even more important than temperature (Wurr et al., 1988). Hand et al. (1992) measured and analyzed the net photosynthesis rate with everyday integrals of solar radiation. A forecasting regression equation of canopy net photosynthesis rate had been gotten. Comparisons of this regression equation with mechanism model of Thornley (1992) showed that light respond curves of the two models were very close.

The objective of this study is to develop a regression cucumber dry matter production model with a minimum number of parameters. Logistic growth equation was selected to build the regression model. Although it is an ideal model for accumulation equation of dry matter (Li, 1996; Liu, 1994), its form is too simple. Because only is time adopted as independent variable, It could not describe the change of crop growth with the change of environmental conditions such as illumination and temperature, etc. Therefore the Logistic growth equation needs to be improved. 


\section{MATERIALS AND METHODS}

Five different tests were carried out for model foundation and verification. Test 1 was for model foundation, and Tests 2-5 for model verification.

\section{$2.1 \quad$ Test 1}

Test 1 was achieved in solar greenhouse in Beijing Agricultural Science Academy from April to July of 2003. Cucumber (Cucumis sativus L.) was named Beijing New No. 4, and the seedlings were transplanted on April 16 of 2003. Pot soilless culture was applied and substrate was mixture of charcoal and vermiculite with the rate of $2: 1(\mathrm{w} / \mathrm{w})$, and drip irrigation was used to deliver nutrient solution to the plants. In the process of development and growth, all axillaries shoots were removed. Test area was $66 \mathrm{~m}^{2}$ and density was 5 plants $/ \mathrm{m}^{2}$.

The environmental data, such as light, temperature, and $\mathrm{CO}_{2}$ concentration were recorded at 10-minute intervals automatically. Six plants were sampled at 10-day (not including roots) intervals, and dry matter weights of leaf, stem, fruit (including flower) and leafstalk were determined. Assumption that root takes 3\% of total dry weight was accepted (Marcelis, 1994).

Methods and contents of sampling and measurement in test 2-5 were the same as test 1 .

\section{$2.2 \quad$ Test 2}

Test 2 was fulfilled in the solar greenhouse in Beijing Agricultural Science Academy from April to July of 2004. The seeds were sown on March 8, 2004, and the seedlings were placed in substrate on April 6, 2004. Cucumber variety and culture type were the same as Test 1, but the difference was that greenhouse was covered with sunshade which transmittance was $35-40 \%$ and $37 \%$ in average.

\subsection{Test 3}

Test 3 was completed in the solar greenhouse in China Agricultural University (Beijing) from September to November of 2004. Cucumber was named Jinglv No. 2 and the seedlings were transplanted in charcoal groove built by bricks. Plant density was 7 plants per square meter. The others of water and fertilizer management were the same as Test 1. 


\section{$2.4 \quad$ Test 4}

Test 4 was gone on in the solar greenhouse in Chinese Academy of Agricultural Sciences (Beijing) from April to July of 2005. The seeds of cucumber, Mini No. 2, were sown on April 3, 2005. The brick grooves were the same as test 3 . Substrate was mixture of corn straw and soil in the rate of 3:1 (w/w). Management of water and fertilizer were not difference from Test 1 .

\section{$2.5 \quad$ Test 5}

Test 5 was accomplished in the solar greenhouse in Chinese Academy of Agricultural Sciences (Beijing) from April to July of 2005. Cucumber variety and culture type were the same as Test 4 . The difference was that greenhouse was covered with sunshade which transmittance was $35-45 \%$ and $40 \%$ in average.

\section{MODEL ESTABLISHMENT}

The independent variable of time in Logistic model was replaced with a function of effective temperature accumulation (ETA) and effective light intensity accumulation (ELIA), so the two main factors for crop growth, temperature and light, was considered.

The equation was:

$$
D M A=\frac{D r_{\max }}{1+e^{\tau(E T A, E L I A)}}
$$

where

$D M A=$ dry matter weight per plant, $\mathrm{g}$

$D r_{\text {max }}=$ the biggest of dry matter weight per plant, $\mathrm{g}$

$E T A=$ effective temperature accumulation, ${ }^{\circ} \mathrm{C} \mathrm{d}$

$E L I A=$ effective light intensity accumulation, $\mu \mathrm{mol} \mathrm{m}{ }^{-2}$

$\tau=$ a function about ETA and ELIA

ETA was defined as the sum of the temperature which was higher than physiological zero point in certain period, and was calculated as the following equation:

$$
E T A=\operatorname{SUM}\left(T_{\text {mean }}-T_{b}\right)
$$


where

$T_{\text {mean }}=$ the average temperature of 2:00, 8: 00, 14:00, 20: 00 in a day, ${ }^{\circ} \mathrm{C}$

$T_{b}=$ the temperature of cucumber physiological zero point, ${ }^{\circ} \mathrm{C}$

ELIA was defined as the sum of the light intensity which was higher than light compensation point multiplied with time in certain period. Photosynthetic photon flux density and illumination length were considered together. The calculating equation was:

$$
R D=S U M\left[L_{t} \times\left(R-R_{b}\right)\right] \quad E L I A=\operatorname{SUM}(R D)
$$

where

$R D=$ the sum of the light intensity which was higher than light compensation point multiplied with time in a day, $\mu \mathrm{mol} \mathrm{m}^{-2}$

$R=$ photosynthetic photon flux density in certain time, $\mu \mathrm{mol} \mathrm{m} \mathrm{m}^{-2}$

$R_{b}=$ light compensation point of cucumber, $51 \mu \mathrm{mol} \mathrm{m}^{-2} \mathrm{~s}^{-1}$

$L_{t}=$ time step, $600 \mathrm{~s}$

Finally, the DMA model based on ETA and ELIA was described as:

$$
D M A=\frac{D r_{\max }}{1+\exp (\varepsilon+\lambda E T A+\omega E L I A)}
$$

\section{MODEL VERIFICATION}

The above-mentioned model was verified by the Test 2-5 data of dry matter weight as well as temperature and light. $D r_{\max }$ of Tests 2 and 3 was $70 \mathrm{~g}$, but $D r_{\max }$ of tests 4 and 5 was $160 \mathrm{~g}$ because of different cucumber variety. The value of regress coefficient $\mathcal{E}, \lambda, \omega$ were calculated from test data.

The simulated values were very near to the actual measurement values, according to their comparison. Generally, root mean square errors (RMSE) value of the model within 15 is excellent. RMSE of the simulated results of four tests were totally smaller than $6 . R^{2}$ of the simulated results of four tests were all 0.99 . F- statistics results indicated that rejective probability of this equation was below 0.01 , i.e. the tenable probability of this regress equation was very notable level. The results of various appraisement indexes, from the all four tests, implied that this model could be applied to simulate accurately the development changes of greenhouse cucumber DMA in certain conditions. 


\section{CONCLUSIONS}

Concept of effective light intensity accumulation (ELIA), the sum of the light intensity that was higher than light compensation point multiplied with time in certain period, has been put forward in this paper. In this way, photosynthetic photon flux density and the length of illumination time were considered and computed as influencing factors of the cucumber DMA. Meanwhile the sensitivity of different crop for light intensity was taken into account.

Logistic equation has been improved. Two environmental factors, temperature and light, have been led into Logistic equation together. A function about ELIA and ETA were applied to replace the time variable. Therefore, the course of cucumber DMA was not only caused by the influence of time change, but also the environmental factors of temperature and illumination.

Compared with mechanism model, regression model parameters were simple and predicting results were more accurate. As tests and data were limited, more test data were necessary in the future to analyze and adjust the coefficients of the model.

\section{ACKNOWLEDGEMENTS}

The author wishes to thank the anonymous referees for their careful reading of the manuscript and their fruitful comments and suggestions.

\section{REFERENCES}

D. W. Hand, G. Clark, M. A. Hannah, J. H. M. Thornley, J. Warren Wilson. Measuring the canopy net photosythnthesis of glasshouse crops. Journal of Experimental Botany, 1992, 43(248): 375-381.

Heuvelink E. Tomato growth and yield: quantitative analysis and synthesis. Wageningen. 1996.

Hugo Challa, Ep Heuvelink. Photosynthesis driven crop growth models for greenhouse cultivation: advances and bottle-necks. Acta Horticulturae, 1996, 417: 9-22.

Katharine B. Perry, Todd C. Wehner. A heat unit accumulation method for predicting cucumber harvest date. Horticultural Technology. 1996, 6(1): 27-30.

Li Lei, Lou Chunheng, Wen Rujing. Study on the Dry matter Accumulation and Distribution with Various Densities in Cotton. Acta Agriculturae Boreali-Occidentalis Sinica 1996, 5 (2): 10-14.

Li Pingping, Wang Duohui, Deng Qingan. A Study of Dynamic Growth and Potential Production Simulation Model of Salad in Greenhouse. Journal of Biomathematics, 1999, 14(1): 77-81. 
Liu Guiru, Zhang Rongzhi, Lu Jianxiang. Study on Grain Filling Characteristics of Wheat Cultivars Tolerant to Drought. Journal of Agricultural University of Hebei. 1994, 17(4): 43-47.

Marcelis L. F. M. A simulation model for dry matter partitioning in cucumber. Ann. Bot 1994. 74: 43-52.

S. Chamont. Modeling dry matter allocation in cucumber crops-competition between fruits and roots. Acta Horticulturae 328, 1993: 195-203.

Thornley J. H. M., Hand D. W., and Waerrn Wilson J. Modelling canopy net photosynthesis of glasshouse row crops and application to cucumber. Journal of Experimental Botany, 1992. 43, 383-391.

Wurr D. C. E., Fellows J. R., Suckling R. F., Crop continuity and prediction of maturity in the crisp lettuce variety Saladin. J. Agric. Sci. 1988. 111, 481-486. 